JOPAT Vol 19(2), 429- 447, July-December, 2020 Edition

ISSN 2636 - 5448. Https://dx.doi.org/10.4314/jopat.v19i2.4

\title{
Evaluation of the Antibacterial Activities of Isolated Bioactive Components from the plant Adenodolichos paniculatus \\ ${ }^{1}$ Onwuliri, A. E, ${ }^{1}$ Kyahar, I. F, ${ }^{2}$ Ehinmidu, J. O and ${ }^{3}$ Oladosu P. O.
}

${ }^{1}$ Dept. of Pharmaceutical Microbiology and Biotechnology, University of Jos, Nigeria. ${ }^{2}$ Dept. of

Pharmaceutics and Pharmaceutical Microbiology, Ahmadu Bello University Zaria, Nigeria and ${ }^{3}$ Dept. of

Microbiology and Biotechnology, National Institute for Pharmaceutical Research and Development, Abuja, Nigeria.

\begin{abstract}
The three bioactive components isolated included: component A (major phytochemicals were Bis (2ethylhexyl) phthalate (16.36 \%), 9,12-Octadecadienoic acid, ethyl ether (14.77\%) and 9.cis., 11.trans.octadecadie noate $(14.77 \%$ ), component B (major phytochemicals were 9,12-Octadecadienal (Linoleic acid) (40.98\%), Octadecanoic acid (Stearic acid) (9.26 \%), Undecanoic acid, 10-bromo- (10bromoudecanoic acid) (9.26\%) and n-Hexadecanoic acid (Palmitic acid) and component C (cis-9octadecenoic acid (Oleic Acid) (30.45\%), Octadecanoic acid (Stearic acid) (17.33\%)). These components isolated from the chloroform fraction of Adenodolichos paniculatus are used by traditional medicinal practitioners for the management of mouth and throat infections. The antibacterial activities against Streptococcus pyogenes, Staphylococcus aureus and Pseudomonas aeruginosa were evaluated using bioautography and agar-well diffusion methods. The bioautogram result showed that component A had inhibited spots against $S$. pyogenes $(17.50 \mathrm{~mm})$ and $P$. aeruginosa $(16.00 \mathrm{~mm})$, corresponding to the TLC spots with Rf values of $0.594,0.55$ and 0.26 , respectively. Component B showed inhibition spots against Streptococcus pyogenes $(36.50 \mathrm{~mm})$, Staphylococcus aureus $(16.00 \mathrm{~mm})$ and Pseudomonas aeruginosa $(11.00 \mathrm{~mm})$, corresponding to the TLC spots with Rf values $0.891,0.87,0.85$ and 0.25 , respectively. Component C showed inhibition spots against Streptococcus pyogenes $(16.50 \mathrm{~mm})$, Staphylococcus aureus $(15.00 \mathrm{~mm})$ and Pseudomonas aeruginosa $(10.50 \mathrm{~mm})$, corresponding to the TLC spots Rf values of 0.938 , 0.44, 0.21 and 0.90, respectively. For the agar-well diffusion method, component A at $1 \mathrm{mg} / \mathrm{ml}$ inhibited Streptococcus pyogenes, Staphylococcus aureus and Pseudomonas aeruginosa growths with zones of inhibition 23.0, 19.5 and $17.50 \mathrm{~mm}$, respectively. MIC and MBC of component A were 125, 250 and 250 and 250, 500 and $500 \mu \mathrm{g} / \mathrm{ml}$, respectively. Component B at $1 \mathrm{mg} / \mathrm{ml}$ inhibited Streptococcus pyogenes, Staphylococcus aureus and Pseudomonas aeruginosa growth with zones of inhibition 30.0, 28.0 and 18.5 $\mathrm{mm}$, respectively. MIC and MBC of the compound B were 31, 62 and 125 and 62, 125 and $250 \mu \mathrm{g} / \mathrm{ml}$, respectively. Component C at $1 \mathrm{mg} / \mathrm{ml}$ inhibited Streptococcus pyogenes, Staphylococcus aureus and Pseudomonas aeruginosa growth with zones of inhibition 24.5, 20.5 and $17.0 \mathrm{~mm}$, respectively. MIC and MBC of the component $\mathrm{C}$ were 62,125 and 250 and 125,250 and $500 \mu \mathrm{g} / \mathrm{ml}$, respectively. This study confirmed that bioactive compounds of A. paniculatus root have antibacterial properties and support the use of this part of the plant as a traditional remedy for mouth and throat infections possibly caused by the test bacteria.
\end{abstract}

Keywords: Adenodolichos paniculatus, isolated compounds, antibacterial activity, pathogenic bacteria, bioautogram, minimum inhibitory concentration (MIC), minimum bactericidal concentration (MBC), Inhibition zone diameter (IZD)

Correspondence: kyaharfriday@yahoo.com

(2)007 The authors. This work is licensed under the Creative Attribution 4.0 International license. 


\section{Journal of Phytomedicine and Therapeutics}

\section{Introduction}

For a long time, plants have been a valuable source of natural products for maintaining human health, with more intensive studies for natural therapies. The use of plant compounds for pharmaceutical purposes has increased in Nigeria. According to the World Health Organization (WHO, 2002), medicinal plants would be the best source to obtain a variety of drugs. About $80 \%$ of individuals from developed countries use traditional medicine, which has compounds derived from medicinal plants. Therefore, such plants should be investigated for a better understanding of their properties, safety and efficiency (Ellof, J.N, 1998). Many plants have been used because of their antimicrobial traits, which are due to compounds synthesized in the secondary metabolism of the plants. These products are known by their active substances; for example, the phenolic compounds which are part of the essential oils as well as tannin (Jansen, Cheffer and Svendsen, 1987). Hence, more studies on the use of plants as therapeutic agents should be emphasized, especially those related to the control of antibiotic-resistant microbes.

Adenodolichos paniculatus (a shrub of 4 - $5 \mathrm{~m}$ high found in the savanna, the bush and the jungle, from Guinea to Northern Nigeria and across to Sudan) is also known in Nigeria by various ethnic names such as kpàrák (Berom), gargung (Mwaghavul), kilikainawa (Fulfulde) and waken wuta (Hausa) to mention but a few. It is one of the important medicinal plants with widespread traditional uses in the remedy and management of mouth and throat infections in Pushit community of Plateau state. Other folkloric uses include dressing of burns, healing of dysentery, liver trouble, venereal diseases, dysentery, diarrhea, and blennorrhoea and also for pain relief (Burkill 1985a; Sani et al., 2010).

The scientific study of $A$. paniculatus has become imperative due to the alarming incidence of antibiotic resistance in bacteria of medical importance and the need to look for other sources of novel antibacterial agents particularly from medicinal plants, based on ethnopharmacological information (Parekh, et al., 2005). Although pharmaceutical industries have produced several new antibiotics in the last three decades, resistance to these drugs by microorganisms has increased. In general, bacteria have the genetic ability to transmit and acquire resistance to drugs, which are utilized as therapeutic agents (Cohen, M.L, 1992). Nascimento et al. (2000) documented a high incidence of resistant microorganisms in clinical microbiology in Brazil. This fact has also been verified in other clinics all over the world (Mulyanningih et al., 2010).

The problem of microbial resistance is growing and the outlook for the use of antimicrobial drugs in the future is still uncertain. Therefore, actions must be taken to reduce this problem; for 


\section{Journal of Phytomedicine and Therapeutics}

Kyahar et al

example, to control the use of antibiotic, increase research to better understand the genetic mechanisms of resistance, and continue studies to develop new drugs, either synthetic or natural. The ultimate goal is to offer appropriate and efficient antimicrobial drugs to the patient.

There has been an effort to document both the phytochemicals present in methanolic leaf extract of A. paniculatus while investigating the analgesic properties of the plant (Sani et al., 2010) in Nigeria. Similar efforts have been made to document the bioactive compounds present in ethyl acetate leaf extract of the plant while studying the isolation, characterization and antimicrobial activity of the plant (Isyaku, Bello and Ndukwe, 2017). To date, there was no literature on the antibacterial activities of isolated bioactive compounds of the root of Adenodolichos paniculatus. It was not also known which component of the root bioactive compounds has antibacterial activity, if any. This study was, therefore, conducted to evaluate the antibacterial susceptibility of Streptococcus pyogenes, Staphylococcus aureus, Pseudomonas aeruginosa and E. coli to the bioactive compounds isolated from the chloroform fractionated components of the root of $A$. paniculatus.

\section{Materials and Methods}

\section{Collection, Identification and Extraction of Plant Materials}

The plant specimens were harvested from the wild plant between October 2018 and March
2019. A herbalist was consulted as his experience was used to determine the accurate location of the plant within the District of Pushit in Mangu LGA of Plateau state, Nigeria. The collected roots were washed with clean water, cut into bits and spread thinly and evenly over an old newspaper and air-dried at room temperature $\left(25^{\circ} \mathrm{C}\right)$. The dried samples were pulverized with a mechanical grinder, sieved with $2.5 \mathrm{~mm}$ sieve and packed into clean, dried bottles and covered tightly. The bottles were labeled with the plant name and date of collection and kept/stored at room temperature until ready for extraction. The plant was identified as Adenodolichos paniculatus on voucher number FHJ 205 and deposited at the Herbarium unit of Federal College of Forestry, Jos. The bioactive constituents of the plant were extracted with five solvent systems (n-hexane, ethyl acetate, chloroform, methanol and water). This was to ensure that compounds with a wide range of polarity could be extracted. The serial maceration extraction technique was employed and successive extraction was carried out with solvents of increasing polarity from non-polar to more polar solvents (Thakare, 2004).

One kilogram $(1 \mathrm{~kg})$ of the powdered sample was extracted in a flat bottom flask with 2.5 liters hexane by maceration for 24 hours with intermittent shaking with an Orbital flask shaker. The sample mixture was filtered twice with muslin cloth, then with vacuum pump filtration and the filtrate was collected. The 


\section{Journal of Phytomedicine and Therapeutics}

Kyahar et al

filtrate was concentrated using a vacuum rotary evaporator at $40{ }^{\circ} \mathrm{C}$. The concentrated/dried extract was collected into a pre-weighed sterile universal bottle, allowed to dry to constant weight at room temperature $\left(25{ }^{\circ} \mathrm{C}\right)$ and weighed. The contents were then weighed for their extract yields and recorded. The bottle was labeled accordingly and stored in a refrigerator for microbial assay. This process was repeated using chloroform, ethyl acetate and methanol after allowing the marc to air dry for 2 to 3 hours. After successive extraction with these solvents, the residue (marc) was collected, air dried (to remove any residue of the solvent) and then macerated with cold distilled water to obtain the aqueous (water) extract. The percentage extract yields of the plant samples were calculated as:

Percentage extract yield $(\%)=$

Weight of dried extract x 100 (Obafemi et al., 2006).

Weight of dried powder

\section{Test Microorganism}

The typed and clinical isolates used for the study included:

Staphylococcus aureus-ATCC6538, Escherichia coli-ATCC43888 and Pseudomonas aeruginosaATCC9027 were obtained from Bacteriology Unit-National Veterinary Research Institute (NVRI), Vom, Plateau state and Streptococcus pyogenes- Clinical isolate obtained Department of Microbiology and Bacteriology, National Institute for Pharmaceutical Research and Development-Diagnostic Centre, Abuja-Nigeria.

The purity of the isolates was ascertained by plating on different selective agar media before carrying out biochemical tests. The purity of the test bacteria was confirmed by sub-culturing into nutrient broth and incubating at $37{ }^{\circ} \mathrm{C}$ for 18 hours. The 18-hour broth culture was streaked unto sterile nutrient agar plates and incubated at $37^{\circ} \mathrm{C}$ for 18 hours. The colonies were observed under the light microscope after simple Gram staining. Isolation of specific bacteria was done by streaking on selective media. A loopful of inoculum from the 18-hour broth culture was streaked on selective agar and incubated at $37{ }^{\circ} \mathrm{C}$ for 18 hours. The cultural characteristics of Escherichia coli were streaked on MacConkey agar to differentiate coliform (pink colony) from non-coliform (non-lactose fermenters) and checked on Eosin Methylene blue agar. Pseudomonas aeruginosa was checked on Cetrimide agar, Staphylococcus aureus on mannitol salt agar and Streptococcus pyogenes on blood agar. The cultural characteristics were observed and compared to standard references (Murray et al, 2007). They were further subjected to various biochemical tests for confirmation.

\section{Standardization of Inocula}

The inoculum was standardized by using the Clinical Laboratory Standard Institute (CLSI, 2000 a) as adapted by Adeshina et al. (2010). 


\section{Journal of Phytomedicine and Therapeutics}

Kyahar et al

Eighteen-hour broth culture of each test organism was standardized by gradually adding normal saline to compare its turbidity to McFarland standard of 0.5 which is approximately $1.0 \times 10^{6} \mathrm{cfu} / \mathrm{ml}$. The turbidity of the cell culture was matched with that of the 0.5 McFarland standard by holding the mixture and the standard in front of light against a white background with contrasting black lines through visual comparison with its density by the addition of normal saline.

\section{Standard McFarland preparation}

The solution of $0.5 \mathrm{ml}$ of $0.048 \mathrm{M} \mathrm{Bacl}_{2}$ was added to $99.5 \mathrm{ml}$ of a $0.18 \mathrm{M} \mathrm{H}_{2} \mathrm{SO}_{4}$ solution and vortex for 2 minutes. A UV-Vis spectrophotometer was used to measure the absorbance of the solution at $625 \mathrm{~nm}$. An absorbance of 0.1 was obtained, which was in the accepted range of 0.08 to 0.13 . This standard (0.5) was used to make a visual comparison with the density of the suspension against a white background with black lines.

\section{Antimicrobial Susceptibility Test}

The agar-well diffusion method of CLSI (2009) as adapted by Sedighinia et al., (2012) was employed to screen the isolated components for antibacterial activity. Overnight broth cultures were diluted appropriately using the McFarland scale (0.5 McFarland which is approximately $\left.1.0 \times 10^{6} \mathrm{cfu} / \mathrm{ml}\right)$. The molten sterile MuellerHinton agar-MHA $(20 \mathrm{ml})$ was poured into a sterile Petri dish and allowed to set. The sterile
MHA plates were flooded with $1.0 \mathrm{ml}$ of standardized culture of each test organism and the excess drained off. Wells of $6 \mathrm{~mm}$ diameter were bored into the inoculated plates using sterile a cork borer $(6 \mathrm{~mm})$. One drop of the molten agar was used to seal the bottom of the bored hole so that the isolated compound would not seep beneath the agar. Ten milligrams (10 $\mathrm{mg}$ ) of each isolated component was dissolved in $2 \mathrm{ml}$ of $10 \%$ dimethyl sulfoxide (DMSO) solution in water to obtain a stock solution of 5 $\mathrm{mg} / \mathrm{ml}$ concentration. Using a micropipette, two hundred microliters $(200 \mu \mathrm{l})$ of the isolated component stock concentration was dispensed into each agar-well. Standard antibiotic (ofloxacin) was used as positive control while sterile distilled water and $10 \%$ DMSO served as negative controls. They were allowed to diffuse into the agar at room temperature for one hour before incubation at $37{ }^{\circ} \mathrm{C}$ for 18 hours. The experiment was carried out in duplicates; the inhibition zone diameters were measured to the nearest millimeter ( $\mathrm{mm})$ and mean and standard deviation of the duplicates were recorded (Ali et al., 2011).

\section{Determination of Minimum Inhibitory Concentration (M.I.C.)}

The agar-well dilution method (modified) according to CLSI (2012) as adapted by Campana et al. (2014) was employed to determine the minimum inhibitory concentration of isolated compounds. Ten milliliters $(10 \mathrm{ml})$ of the various isolated component concentrations 


\section{Journal of Phytomedicine and Therapeutics}

Kyahar et al

prepared in $10 \%$ dimethyl sulfoxide $(2,1,0.5$, $0.25,0.125,0.06$ and $0.031 \mathrm{mg} / \mathrm{ml}$ ) were mixed with $10 \mathrm{ml}$ of double strength Molten MuellerHinton agar (prepared by doubling the manufacturer's recommended weight of the agar to be dissolved in the same volume water) at 45 ${ }^{0} \mathrm{C}$ and poured into Petri-plates aseptically and allowed to solidify. The mixture gave a final various component concentration of $1,0.5$, $0.125,0.0625,0.031,0.0156$ and $0.0078 \mathrm{mg} / \mathrm{ml}$ for the study. After setting, sterile paper discs (6 $\mathrm{mm}$ ) were applied at equidistance to the surface of the set agar containing the various isolated component concentrations. Ten microliters (10 $\mu 1)$ of each standardized organism/inoculum $\left(10^{6} \mathrm{cfu} / \mathrm{ml}\right)$ were then spot-inoculated on each disc and allowed to diffuse for 30 minutes before incubating at $37^{\circ} \mathrm{C}$ for 18 hours. Growth control was prepared by inoculating $10 \mu \mathrm{l}$ of each culture suspension on $15 \mathrm{ml}$ MHA medium without any compound or solvent (drug-free medium). Solvent control was prepared by pouring $1 \mathrm{ml}$ of $10 \%$ DMSO into $14 \mathrm{ml}$ of MHA medium followed by seeding of cultures. The first lowest concentration that showed no visible growth of the inoculated test organism was recorded as the MIC of the isolated compound for the test organism after overnight incubation ((Ali et al., 2011).

\section{Determination of Minimum Bactericidal Concentration (M.B.C.)}

All inoculated paper discs showing no visible growth from MIC determination were aseptically transferred to $5 \mathrm{ml}$ of sterile nutrient broth containing $5 \%$ yeast extract and $3 \%$ Tween 80 to neutralize the effect of the components and incubated for another 18 hours at $37{ }^{\circ} \mathrm{C}$. The discs from the lowest concentration of each isolated compound that showed no visible growth (cloudiness) was taken as the MBC of each plant extract against the test organism.

\section{Bioautographic studies}

For the bioautographic assay, the agar overlay method developed by Fisher and Lautner (1961) as adapted by Choma and Grzelak (2015) and Udobi et al. (2010) was adopted. Ten (10) $\mathrm{ml}$ of molten nutrient agar (NA) was seeded with 0.1 $\mathrm{ml}$ of standardized culture of the susceptible test organisms (Streptococcus pyogenes, Staphylococcus aureus and Pseudomonas aeruginosa) and shaken very well. The seeded molten agar was poured/spread thinly over the developed TLC plates earlier air-dried for 24 hours in Petri-dishes. It was allowed to solidify and pre-diffuse for 2 hours before wrapping the plates with foil paper and incubating at $37{ }^{\circ} \mathrm{C}$ for 24 hours. The plates were then sprayed with an aqueous solution (2 $\mathrm{mg} / \mathrm{ml})$ of methylthiozolyltetrazolium chloride (M.T.T) and further incubated at $37{ }^{\circ} \mathrm{C}$ for 2 hours for detection of dehydrogenase activity. Microbial growth inhibition was observed as clear spots/bands against a purple background. The Rf values of spots/bands showing inhibition were determined. 


\section{Journal of Phytomedicine and Therapeutics}

Isolation of bioactive compounds from the most active fraction

Bioactive spots/bands showing inhibition zones on the bioautogram were further purified using multiple development preparative thin layer chromatography (PTLC). In this step, the preparative glass chromatographic technique was employed for the separation and isolation of the bioactive components. The most active column fraction (A5) was spotted along a horizontal line of the activated glass preparative $(20 \times 20 \mathrm{~cm}$, thickness $0.5 \mathrm{~mm})$ at room temperature. After a proper run of the solvent system (hexane: ethyl acetate, 80:20), the plate was taken out of the chromatographic chamber and air-dried for 24 hours. The plates were visualized under ultraviolet light (254 and 360 $\mathrm{nm})$. The straight-line bands that developed on the plate were scrapped off using a clean razor blade along the sorbent into a conical flask. Chloroform $(10 \mathrm{ml})$ was added and the suspension obtained was left to stand for 30 minutes to facilitate leaching of the compound into the solvent and then filtered. This isolation process was subjected to repeated preparative TLC (X 5) to ensure maximum recovery. The filtrates were left in an open crucible for the chloroform to evaporate. The compounds recovered from the PTLC were weighed and evaluated for antibacterial activity using the agar-well and dilution methods.

\section{Results}

The TLC analysis, characteristics and percentage yields of isolated bioactive components as presented in Table 1 showed that the percentage yield was poor (component A was 7.66, B 7.76 and C $1.21 \%$ ). Component A was brown with a melting point of 133-143, B was light brown with a melting point of 130140 and $\mathrm{C}$ was dark brown with a melting point of 130-142. The Rf values of each component (A, B and C) were 0.594, 0.89 and 0.94 respectively.

Table 1: TLC Analysis, Characteristics and Percentage Yield of Isolated Bioactive Components

\begin{tabular}{lllll}
\hline $\begin{array}{l}\text { Bioactive } \\
\text { component }\end{array}$ & $\begin{array}{l}\text { Percentage } \\
\text { yield }(\%)\end{array}$ & RF value & Description & $\begin{array}{l}\text { Melting point } \\
\left({ }^{\circ} \mathbf{C}\right)\end{array}$ \\
\hline A & 7.66 & 0.594 & Brown powder & $133-143$ \\
B & 7.76 & 0.891 & Light brown powder & $130-140$ \\
C & 1.21 & 0.938 & Dark green powder & $130-142$ \\
\hline
\end{tabular}




\section{Journal of Phytomedicine and Therapeutics}

Table 2: Zone of inhibition of the Bioactive Components in the Pooled Column Fractions from Chloroform Fraction of $A$. paniculatus Root on TLC bioautogram

\begin{tabular}{llll}
\hline Isolated components & \multicolumn{2}{l}{ Test bacteria (zone of inhibition-mm) } \\
\hline & S. pyogenes & S. aureus & P. aeruginosa \\
$A$ & $17.5 \pm 0.71$ & NA & $16.0 \pm 0.00$ \\
B & $36.5 \pm 0.71$ & $16.0 \pm 0.00$ & $11.0 \pm 0.00$ \\
C & $16.5 \pm 0.71$ & $15.0 \pm 0.00$ & $10.5 \pm 0.71$ \\
Rf Value of component A & 0.26 & NA & 0.55 \\
Rf Value of component B & 0.85 & 0.87 & 0.25 \\
Rf Value of component C & 0.9 & 0.44 & 0.21 \\
\hline
\end{tabular}

Values are mean inhibition zone $(\mathrm{mm}) \pm \mathrm{S}$.D of two replicates

NA $=$ No activity

Table 3: Antibacterial Activity (inhibition zone diameter) of the Bioactive Components in the Pooled Column Fraction from Root Chloroform Fraction of $A$. paniculatus.

\begin{tabular}{llll}
\hline Isolated component $(\mathbf{1 ~} \mathbf{~ m g} / \mathbf{m l})$ & \multicolumn{3}{l}{ Test bacteria/ zone of inhibition $(\mathbf{m m})$} \\
\hline & S. pyogenes & S. aureus & P. aeruginosa \\
A & $23.5 \pm 0.71$ & $19.5 \pm 0.71$ & $17.5 \pm 0.710$ \\
B & $30.0 \pm 0.00$ & $28.0 \pm 0.00$ & $18.5 \pm 0.71$ \\
C & $24.5 \pm 0.71$ & $20.5 \pm 0.71$ & $17.0 \pm 0.00$ \\
Ofloxacin $(5 \mu \mathrm{g})$ & 32 & 30 & 31 \\
DMSO $(10 \%)$ & NA & NA & NA \\
\hline
\end{tabular}

Values are mean inhibition zone $(\mathrm{mm}) \pm$ S.D of two replicates

NA $=$ No activity 


\section{Journal of Phytomedicine and Therapeutics}

Table 4: Minimum Inhibitory Concentration (MIC) of Bioactive Components from Chloroform Fraction of the Chloroform Root Extract of A. paniculatus

\begin{tabular}{llll}
\hline \multirow{2}{*}{ Isolated Component } & \multicolumn{3}{l}{ MIC $(\boldsymbol{\mu g} / \mathrm{ml})$} \\
\cline { 2 - 4 } & Strep & Staph & Pseudo \\
\hline A & 125 & 250 & 250 \\
B & 31 & 62 & 125 \\
C & 62 & 125 & 250 \\
Ofloxacin & 10 & 10 & 10 \\
DMSO $(10 \%)$ & NA & NA & NA \\
\hline
\end{tabular}

$\mathrm{NA}=$ No activity

Table 5: Minimum Bactericidal Concentration (MBC) of Bioactive Components from Chloroform Fraction of the Chloroform Root Extract of A. paniculatus

\begin{tabular}{llll}
\hline \multirow{2}{*}{ Isolated Component } & \multicolumn{2}{l}{ MBC $(\boldsymbol{\mu g} / \mathrm{ml})$} \\
\cline { 2 - 4 } & Strep & Staph & Pseudo \\
\hline A & 250 & 500 & 500 \\
B & 62 & 125 & 250 \\
C & 125 & 250 & 500 \\
Ofloxacin $(\mu \mathrm{g} / \mathrm{ml})$ & 10 & 10 & 10 \\
DMSO $(10 \%)$ & NA & NA & NA \\
\hline NA = No activity & & &
\end{tabular}




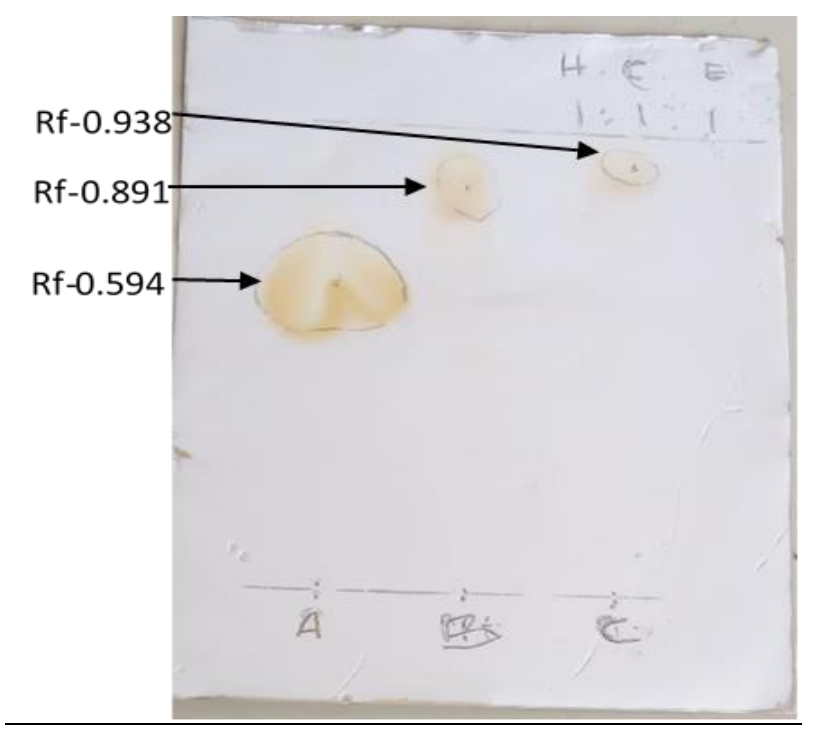

Plate 1: Thin layer chromatogram of the bioactive components showing three spots $(A, B$ and $C)$ in the chloroform fraction from chloroform root extract of $A$. paniculatus

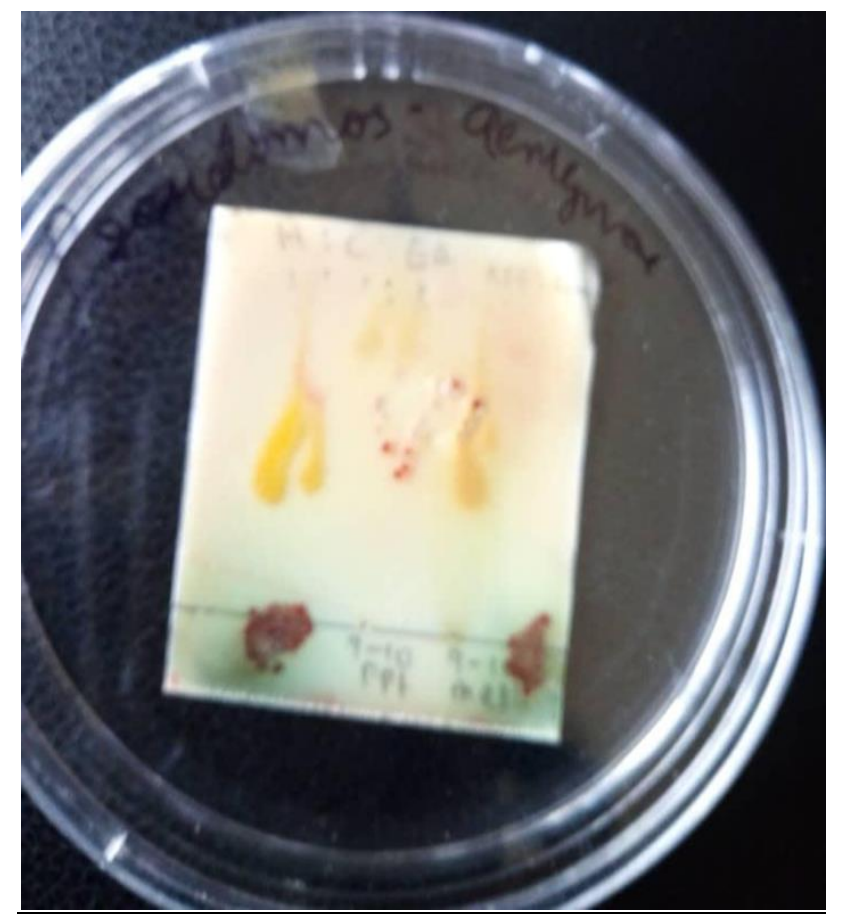

2. Bioautogram of bioactive compounds in the chloroform fraction against Pseudomonas aeruginosa 


\section{Journal of Phytomedicine and Therapeutics}

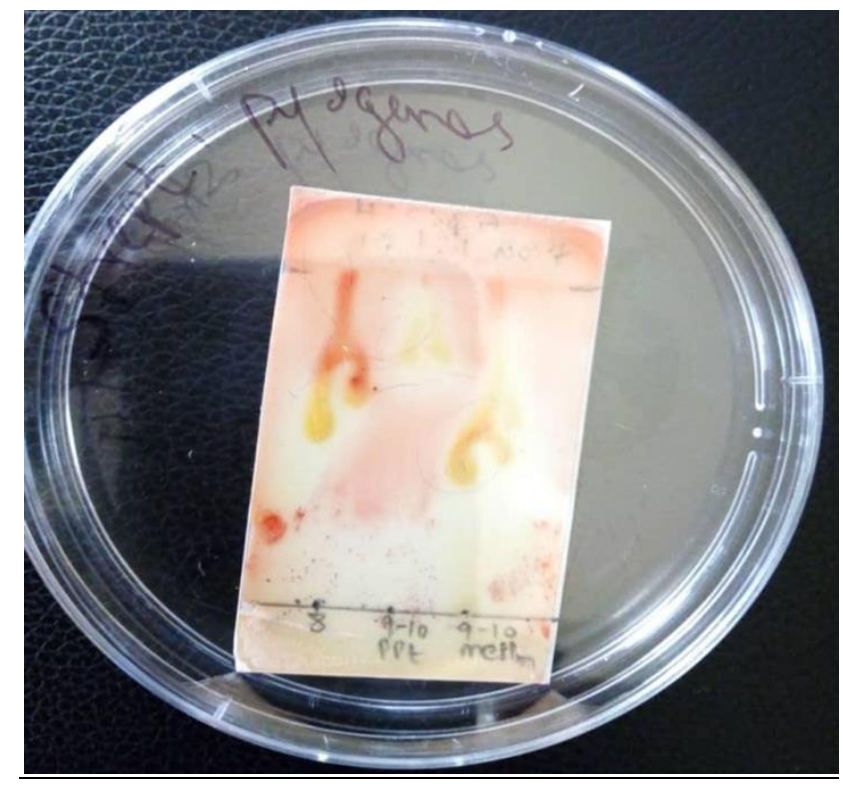

3: Bioautogram of bioactive compounds in chloroform fraction against Streptococcus pyogenes

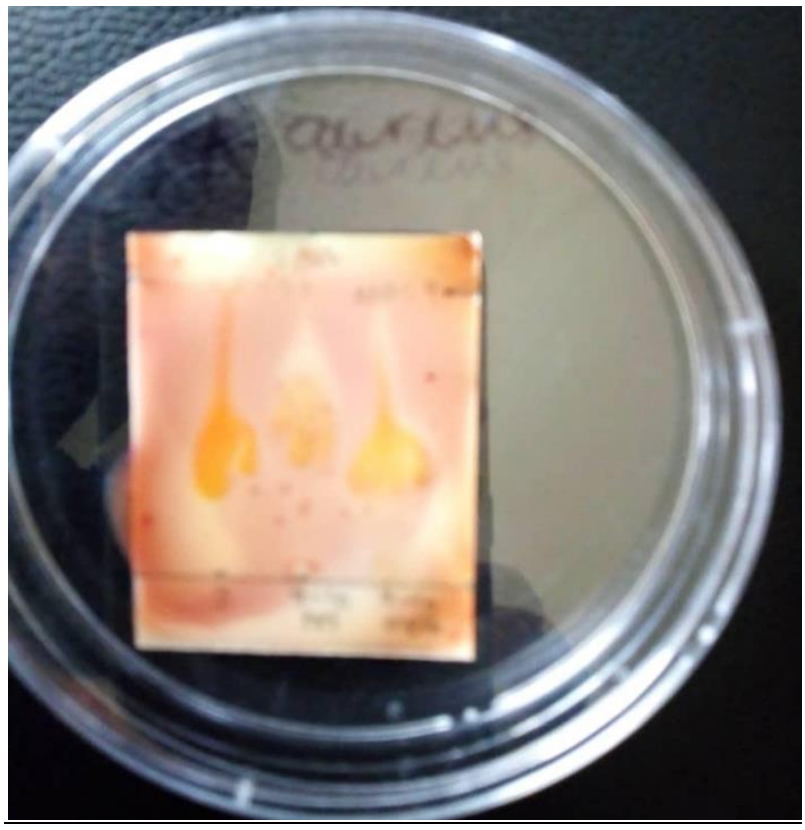

4: Bioautogram of bioactive compounds in chloroform fraction against Staphylococcus aureus 


\section{Journal of Phytomedicine and Therapeutics}

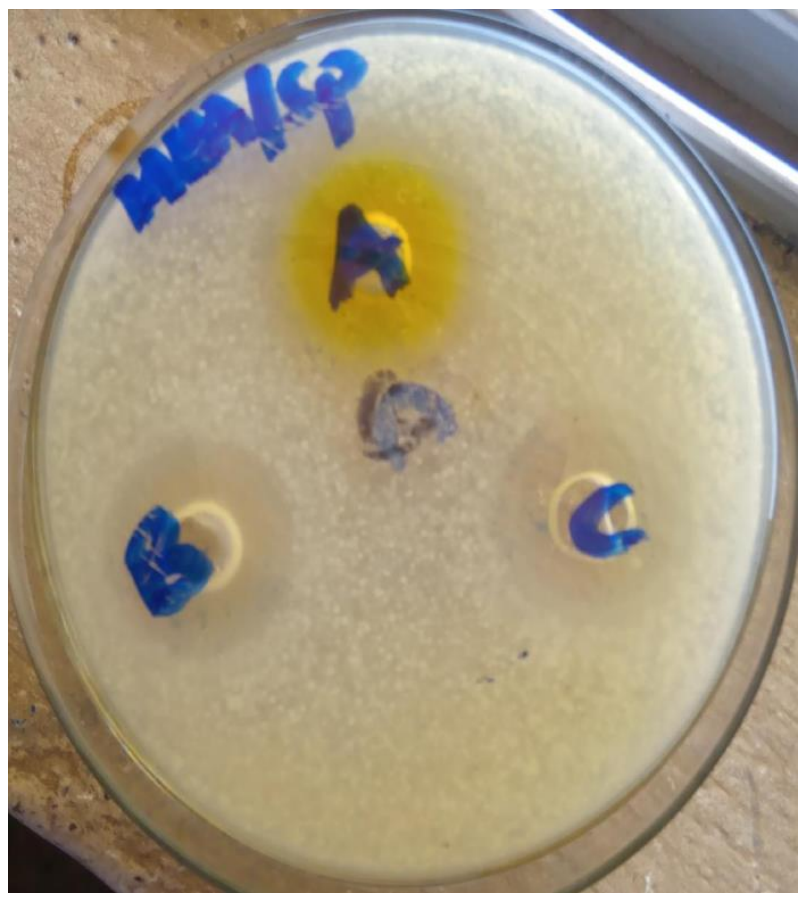

1: Sensitivity of Streptococcus pyogenes to isolated bioactive components A, B and C

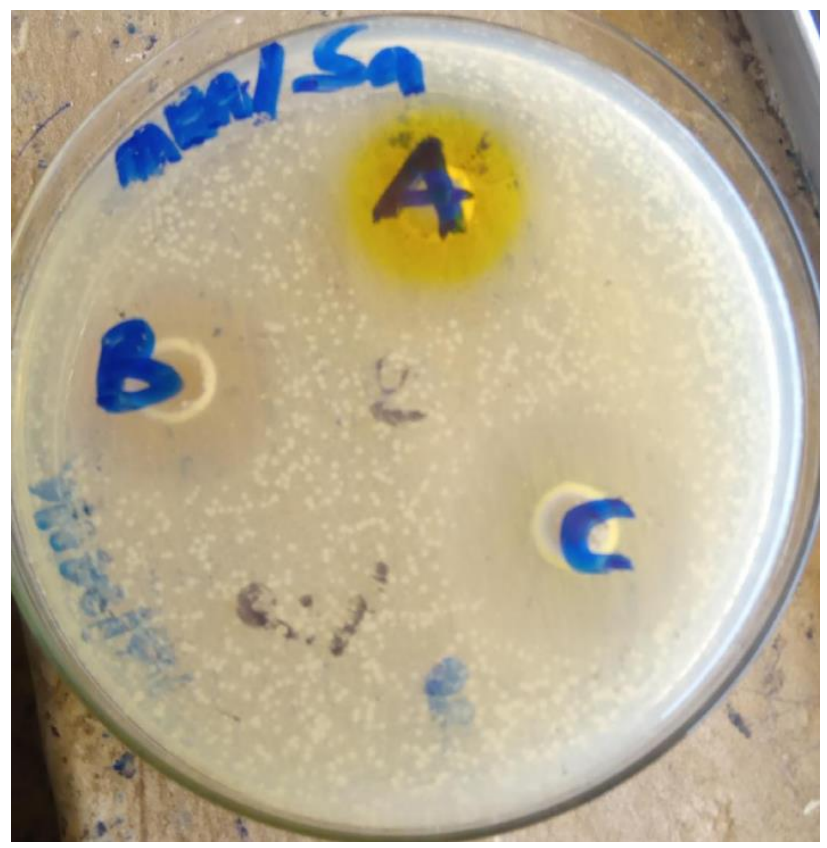

2: Sensitivity of Staphylococcus aureus to isolated bioactive components A, B and C 


\section{Journal of Phytomedicine and Therapeutics}

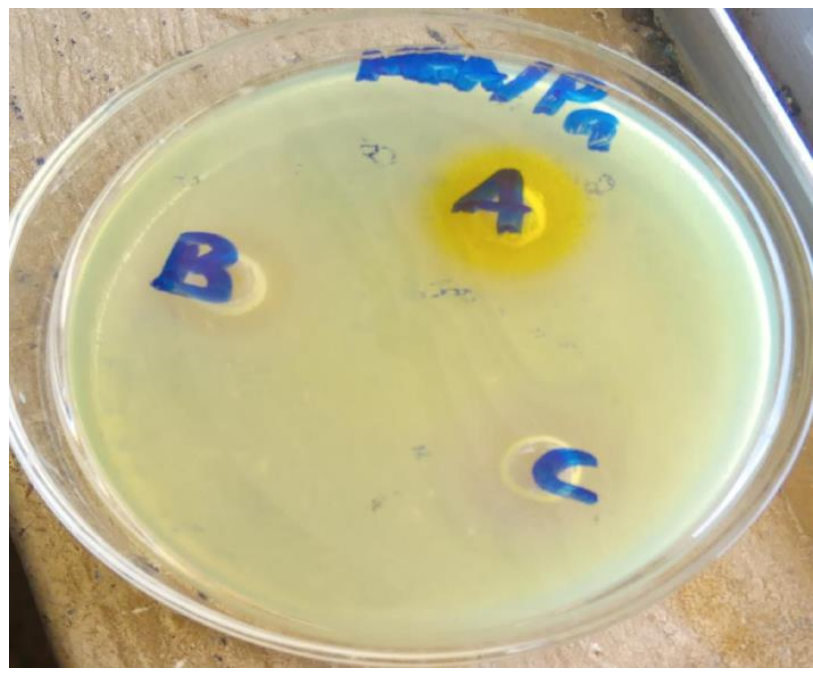

3: Sensitivity of Pseudomonas aeruginosa to isolated bioactive components A, B and C

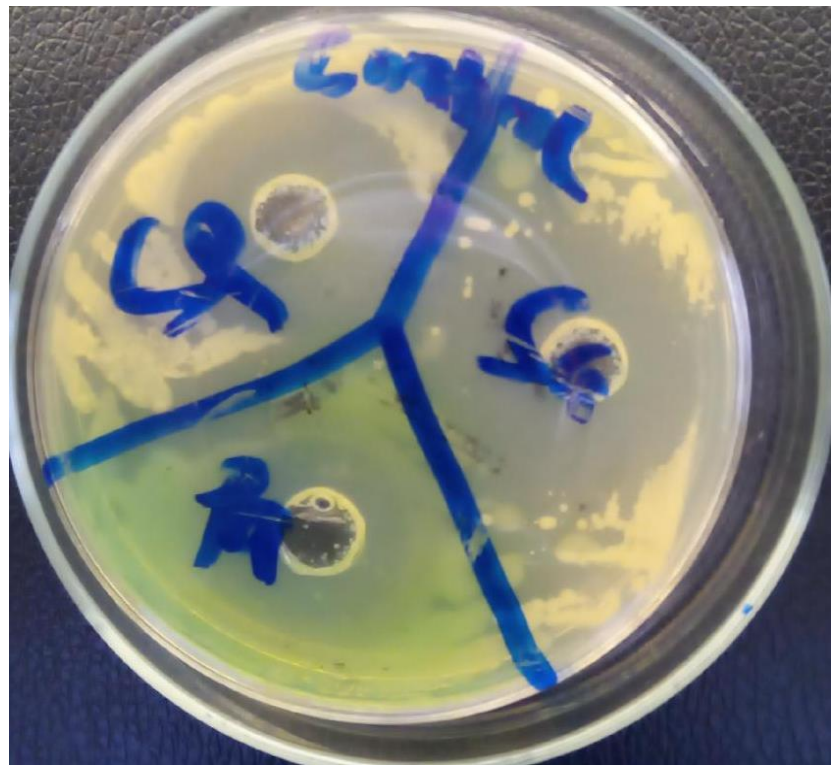

4: Sensitivity of Streptococcus pyogenes, Staphylococcus aureus and Pseudomonas aeruginosa to the referenced antibiotic-Ofloxacin as positive control 


\section{Journal of Phytomedicine and Therapeutics}

Kyahar et al

\section{Discussion}

The result of the TLC analysis showed that the percentage yield of the fraction was poor with the highest at only $7.76 \%$. The result of the study showed that the chloroform root extract exhibited in vitro antibacterial activity on three of the four selected test bacteria at a concentration of $100 \mathrm{mg} / \mathrm{ml}$. The chloroform root extract was further subjected to vacuum liquid chromatography (VLC). The VLC separation of the chloroform extract yielded several fractions and a total of 14 fractions which pooled together based on their TLC characteristics into six combined fractions. The result of the antibacterial in vitro study of the six combined fractions indicated that only two combined fractions (A4 and A5 fractions) at $20 \mathrm{mg} / \mathrm{ml}$ were active against only three (3) of the four (4) pathogens tested; namely, Streptococcus pyogenes, Staphylococcus aureus and Pseudomonas aeruginosa and $E$. coli with zones of inhibition measuring 13.0, 10.5 and $10.00 \mathrm{~mm}$ (A4) and 28.5, 23.0 and $13.5 \mathrm{~mm}$ (A5), respectively. It was not active on E. coli.

The combination fraction (A5), which showed better antibacterial activity, was subjected to a bioautography study. The result showed the appearance of white areas against a purple-red background on the chromatograms, denoting inhibition of growth of the bacteria (Figure 1-3), which was due to the presence of compound(s) that inhibit their growth. The zone of inhibition spots/bands exhibited the isolated bioactive components presented in Table 2 were 17.5 $\mathrm{mm}$ and $16.0 \mathrm{~mm}$ for bioactive component $\mathrm{A}$ against Streptococcus pyogenes and Pseudomonas aeruginosa, respectively. The absence of antibacterial activities against Staphylococcus aureus could be due to evaporation of the active compound (photooxidation) or due to very little amount of the active compound (Masoko and Eloff, 2005). Bioactive component $\mathrm{B}$ has inhibition of 36.5 $\mathrm{mm}, \quad 16.0 \mathrm{~mm}$ and $11.0 \mathrm{~mm}$ against Streptococcus pyogenes, Staphylococcus aureus and Pseudomonas aeruginosa, respectively, while component $\mathrm{C}$ showed $16.5 \mathrm{~mm}, 15.0 \mathrm{~mm}$ and $10.5 \mathrm{~mm}$ for the same organisms, respectively. The $\mathrm{R}_{\mathrm{f}}$ values of the bioactive component A corresponding to the inhibition zones/bands on Streptococcus pyogenes and Pseudomonas aeruginosa were 0.26 and 0.55 , respectively, and no inhibition of microbial growth was observed on Staphylococcus aureus. The $\mathrm{R}_{\mathrm{f}}$ values of the bioactive component $\mathrm{B}$ corresponding to the inhibition zones/bands on Streptococcus pyogenes, Staphylococcus aureus and Pseudomonas aeruginosa were 0.85, 0.87 and 0.25 , respectively. The $R_{f}$ values of the bioactive component $\mathrm{C}$ corresponding to the inhibition zones/bands on Streptococcus pyogenes, Staphylococcus aureus and Pseudomonas aeruginosa were 0.90, 0.44 and 0.21 , respectively. It is possible that 


\section{Journal of Phytomedicine and Therapeutics}

Kyahar et al

synergism played a major role in extracts that were active when the minimum inhibitory concentration of the mixture was determined, but the separated compounds had no antimicrobial activity based on bioautography (Eloff et al., 2011).

Actively growing microorganisms can reduce methylthiozolyltetrazolium chloride (M.T.T) to a purple-red color (Begue and Klein, 1972). In the presence of active plant compounds on the chromatograms, the growth of the organism was inhibited. Because the $R_{\mathrm{f}}$ value is constant for the same compound under defined conditions, the presence of clear bands with the same Rf value may mean that the same compounds are probably responsible for the antimicrobial activity in the same bioactive component tested against the test bacteria. This would suggest a non-selective antimicrobial activity.

Each isolated component exhibited in vitro antibacterial activity on the three sensitive test bacteria at a concentration of $1 \mathrm{mg} / \mathrm{ml}$ as presented in Table 3. Bioactive component A showed antibacterial activity against the three test bacteria ( $S$. aureus, $S$. pyogenes and $P$. aeruginosa) with inhibition zone diameters ranging from 17.5 to $23.5 \mathrm{~mm}$. Bioactive component B showed antibacterial activity against the three test bacteria $(S$. aureus, S. pyogenes and P. aeruginosa) with inhibition zone diameters ranging from 18.5 to $30.0 \mathrm{~mm}$. Bioactive component $\mathrm{C}$ showed antibacterial activity against the three test bacteria ( $S$. aureus, $S$. pyogenes and $P$. aeruginosa) with inhibition zone diameters ranging from 17.0 to $24.5 \mathrm{~mm}$. The largest inhibition zone diameter (30 $\mathrm{mm})$ was produced by bioactive component $\mathrm{B}$ against $S$. pyogenes, $S$. aureus $(28 \mathrm{~mm})$ and $P$. aeruginosa (18.5 $\mathrm{mm})$.The minimum inhibitory concentration of the component A against $S$. pyogenes, $S$. aureus and $P$. aeruginosa were 125, 250 and $250 \mu \mathrm{g} / \mathrm{ml}$, respectively, while the minimum bactericidal concentrations for the three test bacteria were 250,500 and $500 \mu \mathrm{g} / \mathrm{ml}$. The minimum inhibitory concentration of component B against $S$. pyogenes, $S$. aureus and $P$. aeruginosa were 31,62 and 125 $\mu \mathrm{g} / \mathrm{ml}$, respectively, while the minimum bactericidal concentrations for the three test bacteria were 62,125 and $250 \mu \mathrm{g} / \mathrm{ml}$. The minimum inhibitory concentration of component $\mathrm{C}$ against $S$. pyogenes, $S$. aureus and $P$. aeruginosa were 62,125 and 250 $\mu \mathrm{g} / \mathrm{ml}$, respectively, while the minimum bactericidal concentrations for the three test bacteria were 125,250 and $500 \mu \mathrm{g} / \mathrm{ml}$ as presented in tables 4 and 5 respectively. The inhibitory values of the isolated components were much lower (the lowest was $31 \mu \mathrm{g} / \mathrm{ml}$ ) than those of both the extract and fractions (310 and 1,560 $\mu \mathrm{g} / \mathrm{ml}$ ). The MIC and MBC values of each the components against the test organisms were higher than the 


\section{Journal of Phytomedicine and Therapeutics}

Kyahar et al

control/standard drug. This is expected because the compounds may not be $100 \%$ pure, unlike the control dug which is synthetic and $100 \%$ pure.

Bioactive component B produced the highest zones of inhibition against the test bacteria, followed by bioactive component $\mathrm{C}$, and then bioactive component $\mathrm{A}$ which did not show any activities on $S$. aureus. The superiority of activities of bioactive component B could mean that the component contained more potent antimicrobial compounds acting synergistically or that the component contained higher concentrations of the bioactive components.

\section{Conclusion}

The three bioactive isolated compounds from A. paniculatus root showed antibacterial activities against $S$. pyogenes, $S$. aureus and $P$. aeruginosa. The diameters of the zones of inhibition of the most active component B were $30.00,28.00$ and $18.5 \mathrm{~mm}$, respectively. The inhibitory effect of bioactive component $B$ against the test bacteria was not statistically effective compared to the standard antibiotic (Ofloxacin) disc, which had the inhibitory zone diameters of 32.00 , 30.00 and $31.00 \mathrm{~mm}$, respectively. The inhibitory activities of the isolated bioactive compounds against the test organisms provide scientific support for the traditional uses of the plant for the treatment of mouth and throat infections caused by any of the test bacteria. It also confirmed that the root of $A$. paniculatus contained bioactive compounds that had an inhibitory effect on the test bacteria. Further investigation will be required to characterize bioactive component $\mathrm{B}$ which showed the most potent antibacterial activity. This may serve as a template for drug discovery. Also, an investigation can be performed to isolate a single bioactive compound to see if the chemical structure can be modified to improve its antibacterial activity.

\section{REFERENCES}

Adeshina, G. O., Onaolapo, J. A., Ehinmidu, J. O and Odama, L. E. (2010). Phytochemical and antimicrobial studies on the ethyl acetate extract of Alchornea cordifolia leaf found in Abuja-

Nigeria. Journal of Medicinal Plants Resources, 4(8), 649-658.

Agilent, (2013). Agilent Cary 630 FTIR operation manual, First ed., Agilent Technologies Inc. Wilmington, DE 198081610 USA.

Agilent, (2013). Agilent 7890B Gas

Chromatography Operation

Manual, First ed., Agilent

Technologies Inc. 


\section{Journal of Phytomedicine and Therapeutics}

Wilmington, DE 19808-1610

USA.

Ali, A.I., Ehinmidu J.O. and Ibrahim

Y.K.E. (2011). Preliminary

phytochemical screening and

antimicrobial activities of some

medicinal plants used in Ebira

land. Bayero Journal of Pure and

Applied Sciences, 4(1), 10-16.

Begue W. J. and Klein, R. M. (1972).

The use of tetrazolium salts

in bioautographic

procedure. Journal of

Chromatography, 88, 182-

184.

Burkill, H. M. (1985a). The Useful Plants of Tropical West Africa, Families A D Royal Botanical Garden, Kew, pp. $1-254$.

Campana, E. H., Carvalhaes, C. G., Nonato,

B., Machado, A. M and Gales, A.

C. (2014). Comparison of M.I.C.E and $E$ test with CLSI agar dilution for antimicrobial susceptibility testing against oxacillin-resistant Staphylococcus spp. PLos ONE, 9(4).

doi.org/10.1371/journal.pone.00946 27.

Choma I.M. and Grzelak E.M. (2011).

Bioautography detection in thin- layer chromatography. Journal of

Chromatography A, 1218, 2684-

2691

Clinical Laboratory Standards Institute

(CLSI) (2009). Methods for

dilution antimicrobial susceptibility

test for bacteria that grow

aerobically-approved guideline M7-

A8. PA, USA: Clinical Laboratory

Standards Institute

Clinical Laboratory Standards Institute

(CLSI) (2012). Performance

standards for antimicrobial

susceptibility testing: twenty-second

Information Supplement (M100-

S22). PA, USA: Clinical

Laboratory Standards Institute.

Cohen, M.L. (1992). Epidemiology of drug resistance: implications

for a post-antimicrobial

era. Science, 257, 1050-

1055.

Chidume, F., Gamaniel, K., Amos, S.,

Akah, P., Obodozie, $\mathrm{O}$ and

Wambebe, C. (2001).

Pharmacological activity of methanolic extract of Cassia nigricans leaves. Indian Journal of Pharmacology, 33(5), 350-356.

Retrieved from

http://medind.nic.in/ibi/t01/i5/ibit01

i5p350.pdf 


\section{Journal of Phytomedicine and Therapeutics}

Dilika F., Bremner P.D. and Meyer J.J. (2000). Antibacterial activity of linoleic and oleic acids isolated from Helichrysum pedunculatun: a plant used during circumcision rites. Fitoterapia, 71(14):450-452. DOI: 1016/s0367-326x (00)-150-7

Ellof, J.N (1998). Which extractant should be used for the screening and isolation of antimicrobial components from plants? Journal of Ethnopharmacology, 60, 1-6.

Eloff, J. N., Ntloedibe, D. T. and Brummelen, R. (2011). A simplified but effective method for the quality control of medicinal plants by planar chromatography. African Journal Traditional Complement Alternative Medicine, 8(S), 1-12.

Jansen, A.M., Cheffer, J.J.C. and Svendsen, A.B. (1987). Antimicrobial activity of essential oils: a 1976-1986 literature review. Aspects of test methods. Planta Med. 40, 395-398.

Murray, P. R., Baron, E. J., Jorgensen, J. H., Landry, M. L. and Pfaller, M.
A. (ed.) (2007). Manual of Clinical Microbiology, 9th Edition. ASM Press, Washington, D. C.

Nascimento G. F., Locatelli, J., Freitas, P... C and Silva G. L. (2000). Antibacterial activity of plant extracts and phytochemicals on antibiotic resistant bacteria. Brazilian Journal of Microbiology, 3, 247-256.

Parekh, J., Jadeja, D. and Chanda, S. (2005). Efficacy of aqueous and methanol extract of some medicinal plants for potential antibacterial activity. Turkish Journal of Biology, 29,203-214.

Sani, M., Anuka, J., Magaji, I., Yaro, A., Magaji, M. and Sani, Y. (2010). Evaluation of analgesic and antiinflammatory activities of the methanolic leaf extract of Adenodolichos paniculatus (hua). Nigerian Journal of Pharmaceutical Sciences, 9(1), $73-80$.

Sedighinia, F., Safipour, A. A., Soleimanpour, S., Zarif, R., Asili, J.and Ghazvini, K. (2012). Antibacterial activity of Glycyrrhiza glabra against oral pathogens: an in-vitro study.

Avicenna Journal of Phytomedicine, 2(3), 118-24. 


\section{Journal of Phytomedicine and Therapeutics}

Thakare, M. (2004). Pharmacological

Screening of Some Medicinal

Plants as Antimicrobial and Feed

Additive. Masters' Thesis.

Department of Animal and Poultry

Science, Virginia Polytechnic

Institute and State University,

Blacksburg, Virginia, USA.

Udobi, C.E., Onaolapo, J. A and Agunu, A. (2008). Antibacterial activities and bioactive components of the aqueous fraction of the stem bark of Parkia biglobosa. Nigerian Journal of Pharmaceutical Sciences, 7 (1) 49-55.

World Health Organization. (2002). Traditional Medicine Strategy20022005 